\title{
Hämatologische Variablen und Plasmacortisol bei Milchrindern in Laufstallhaltung nach der Kalbung und bei deren Kälbern. Effekte einiger konstanter und variabler Faktoren
}

\begin{abstract}
Summary
Title of the paper: Hematological variables and plasma cortisol in dairy cattle kept loose house and in their newborn calves. Effects of some constant and variable factors

On 80 dairy cows (72 Holstein Friesian, 8 German Red and White, kept loose house) and on their newborn calves hemoglobin $(\mathrm{Hb})$, hematocrite (Hk), mean corpuscular hemoglobin concentration (MCHC), plasma cortisol and body temperature (RT) and in calves saliva cortisol and body weight (KM) measurements were done and analysed considering lactation number (LANR) and gestation length (TD) of the cow, sex and KM of the calf, day time and type of birth (GV) and the time between parturition and measurement (ZGM). Type of birth was specified as GV1 (without assistance), GV2 (with moderate assistance by the herdsman, in some cases with mechanical support), and GV 3 (with heavy traction, most with mechanical support). The mean value of $\mathrm{Hb}$ and of MCHC was smaller and that of plasma cortisol was greater in calves comparing dams. In GV2 and GV3 the number of male calves was two times that of female calves. Mean $\mathrm{Hb}$ and Hk of cows were greater in GV2 comparing GV1 and GV3 but this difference was only significant for Hk between GV1 and GV2. In calves $\mathrm{Hb}, \mathrm{Hk}$ and MCHC values became smaller from GV1 to GV3. Mean values of the differences calf-cow of Hb and Hk were significantly different between GV1, GV2 and GV3. Cows and calves with GV1 had significant smaller plasma cortisol concentrations than those with GV2 and GV3. Significant effects on calf plasma cortisol could be established for ZGM, GV, $\mathrm{pCO}_{2}$ and $\mathrm{pH}$ group and those on plasma cortisol of cows for ZGM and $\mathrm{pCO}_{2}$ of calf blood. Saliva cortisol had a stronger correlation with plasma cortisol in calves of group GV1 $(\mathrm{r}=$ $0.749)$ than in those calves of group GV2 ( $r=0.432)$. The Q-Q Plots of the day time of birth and plasma cortisol concentration confirmed effects of the type of birth suggesting a role for diurnal effects. Results show effects of birth assistance measures common used in dairy cattle production on the variation of physiological variables in cows and calves and they were discussed regarding development quality and the reactivity of calves as affected by delivery.
\end{abstract}

Key Words: plasma cortisol, saliva cortisol, hematological variables, loose housed dairy cattle, newborn calves, type of birth

\section{Zusammenfasssung}

An 80 Milchkühen (72 Holstein Friesian, 8 Deutsche Rotbunte, Laufstallhaltung) und deren neugeborenen Kälbern wurden Messungen der Hämoglobinkonzentrationen (Hb), des Hämatokrit (Hk), der mittleren korpuskulären Hämoglobinkonzentration (MCHC), der Plasmacortisolkonzentrationen, des Säure-Basen-Status und der Körpertemperatur (RT), bei Kälbern auch der Speichelcortisolkonzentration und Körpermasse (KM) vorgenommen. Diese wurden unter Berücksichtigung von Laktationsnummer (LANR) und Trächtigkeitsdauer (TD) der Kuh sowie von Geschlecht und KM des Kalbes, Tageszeit der Geburt, Geburtsverlauf (GV) und Zeit zwischen Geburt und Messung (ZGM) analysiert. Die Geburtsverläufe sind als GV1 (ohne Hilfe), GV2 (leichte Zughilfe, in einigen Fällen mit mechanischem Geburtshelfer) und GV 3 (starke Zughilfe, meistens mit mechanischem Geburtshelfer) bezeichnet worden. Hb und MCHC waren bei Kälbern signifikant kleiner und Plasmacortisol signifikant größer als bei Kühen. In GV2 und GV3 war die Anzahl männlicher Kälber doppelt so groß wie diejenige weiblicher Kälber. Die Mittelwerte von Hb und Hk waren bei Kühen mit GV2 größer, sicher waren die Unterschiede von Hk zwischen GV1 und GV2. Bei Kälbern wurden Hb, Hk und MCHC von GV1 nach GV3 kleiner, sicher war der Mittelwertunterschied von Hb zwischen GV1 und GV3. Die mittleren Differenzen KalbKuh von Hb, Hk waren signifikant verschieden zwischen GV1 sowie GV2 und GV3. Kühe und Kälber mit GV1 hatten signifikant kleinere Plasmacortisolwerte als jene mit GV2 und GV3. Sichere Effekte auf Plasmacortisol des Kalbes konnten für ZGM, GV, $\mathrm{pCO}_{2}$ und für die Gruppen nach dem pH-Wert des Blutes und auf Plasma- 
cortisol der Kuh für ZGM und für $\mathrm{pCO}_{2}$ des Blutes des Kalbes nachgewiesen werden. Speichelcortisol hatte eine engere Beziehung zu Plasmacortisol der Kälber in GV1 $(r=0,749)$ als bei jenen in GV2 $(r=0,432)$. Die Q-Q Plots der Tageszeit der Geburt und der Cortisolkonzentrationen bestätigten Effekte durch den Geburtsverlauf und deuteten Einflüsse der Tageszeit an. Die Befunde zeigen, dass in der Milchrindhaltung vom Betreuungspersonal vorgenommene geburtshilfliche Maßnahmen an der Variation physiologischer Variablen der Kühe und der neugeborenen Kälber beteiligt sind, und sie werden unter dem Gesichtspunkt der Entwicklungsqualität und der durch den Geburtsverlauf beeinflussten Reaktionsfähigkeit der Kälber diskutiert.

Schlüsselwörter: Plasmacortisol, Speichelcortisol, hämatologische Variablen, Milchrinder, Laufstallhaltung, neugeborene Kälber, Geburtstyp

1. Einleitung

Die Entwicklungsqualität und auch die Reaktionsfähigkeit der Kälber nach der Geburt beeinflussen die Anpassungsfähigkeit und damit auch die Aufzuchtleistung der Tiere und bestimmen in erheblichem Maße den Betreuungsaufwand während der frühen Aufzuchtperiode. Es ist von Interesse, welche Auswirkungen die vom Betreuungspersonal vorgenommenen geburtshilflichen Maßnahmen in gebräuchlichen Milchrindhaltungen (Laufstallhaltung) auf physiologische Variablen der Kälber haben können. Hämatologische Konzentrationsmaße (Hämoglobin, Hb; Hämatokrit, Hk) waren bei neugeborenen Kälbern kleiner als bei Kühen (KÜHNE, 1986; STEINHARDT et al., 1994b, 1996) und wiesen, wie auch die Plasmacortisolwerte, größere Variationen auf. Übereinstimmend wurden bei Kühen und besonders bei Feten bzw. Kälbern im peripartalen Zeitraum größere Cortisolkonzentrationen festgestellt (EBERHART und PRATT, 1971; GRÜNBERG, 1996; HUNTER et al., 1977; HYDBRING et al., 1999; MASSIP, 1980a,b; STEINHARDT et al., 1996; TAKEISHI et al., 1989). Einflüsse durch die Bedingungen vor der Geburt (BRAASTAD, 1998; LAY et al., 1997) und solche durch den Geburtsverlauf an einem vorwiegend klinisch relevantem Tiermaterial aus verschiedenen Herkünften (HEUWIESER et al. 1987;HOYER et al., 1990; MASSIP 1980a, b; NAKAO und GRUNERT, 1989, 1990) konnten für die Cortisolkonzentration aufgezeigt werden. Unter praktischen betrieblichen Bedingungen sind Effekte des Geburtsverlaufes auf hämatologische Variablen gezeigt (STEINHARDT et al., 1995; 1996), solche auf die Plasmacortisolkonzentration nicht bestätigt worden (BELLOWS und LAMMOGLIA, 2000; GRÜNBERG, 1996; STEINHARDT et al., 1995). Ein Konstantbleiben oder sogar ein Anstieg der Cortisolwerte innerhalb der ersten Stunden nach der Geburt (HOYER et al., 1990; WRUTNIAK und CABELLO, 1987) sowie der stärkere Konzentrationsabfall des Cortisols bei Neugeborenen (EBERHART und PRATT, 1971; STOTT und REINHARD, 1978) und bei Kühen (HEUWIESER et al., 1987) in den ersten $12 \mathrm{~h}$ lässt die Bedeutung der Lebensbedingungen in dieser Zeit und des Zeitpunktes der Messung nach der Geburt erkennen. Die Tageszeit der Geburt ist hinsichtlich der physiologischen Variablen bisher anscheinend nicht berücksichtigt worden. In der vorliegenden Arbeit wurden an einem größeren Tierbestand Einflüsse durch Alter (Laktationsnummer, LANR) und Trächtigkeitsdauer (TD) der Milchkuh, Geschlecht und Körpermasse (KM) des Kalbes, Geburtsverlauf (GV), Tageszeit der Geburt und Zeit zwischen Geburt und Messung (ZGM) auf die Variablen der Tiere bei Laufstallhaltung der Milchkühe und betriebsspezifischer Organisation und Kontrolle der Abkalbung untersucht. Folgende Fragen waren von Interesse: (1) Läßt sich ein Effekt geburtshilflicher Maßnahmen in gebräuchlichen Milchrindhaltungen auf die Plasmacortisolkonzentration der Kühe und der neugeborenen Kälber und auf die Speichelcortisolkonzentration nachweisen? (2) Welche Quali- 
tät haben die Beziehungen physiologischer Variablen der Kühe und der Kälber nach der Geburt?

\section{2. $\quad$ Material und Methoden}

Untersuchte Tiere, Haltungsbedingungen: Tiere der Milchrindherde (72 Deutsche Holstein Friesian, 8 Deutsche Rotbunte) des Institutes für Tierzucht und Tierverhalten waren nach der Weideperiode 1996 (Mai bis September, Dauergrünland, Umtriebsweide, Leckschalen für die Mineralstoffversorgung) in einem Boxenlaufstall (ca. 80 Tiere) aufgestallt worden. Die Winterfütterung bestand aus Silage (Gras- und Maissilage), Konzentratfutter und geringen Mengen von Heu. Auf einem zentralen Futtergang wurde täglich zweimal Futter vorgelegt. Leckschalen standen für die Mineralstoffversorgung zur Verfügung. Außerdem konnten die Tiere von dem täglich frisch eingestreuten Stroh aufnehmen. Die Konzentratfuttergabe erfolgte während des Aufenthaltes der Kühe im Melkstand. Einige Tage vor der voraussichtlichen Abkalbung kamen die Kühe in Abkalbeboxen mit Stroheinstreu (5 Boxen). Nach der Kalbung wurden die Kühe wieder in die Herde und die Kälber in ein Aufzuchtsystem eingegliedert. Die Aufnahme von Kolostrum durch die Kälber aus dem Euter des Muttertieres war möglich, der überwiegende Anteil der Kälber ist jedoch vor der Kolostrumaufnahme untersucht worden. Die Kontrolle der Abkalbungen, geburtshilfliche Maßnahmen und Protokollierungen sind vom Betreuungspersonal in gewohnter Art vorgenommen worden. Die Geburtsverläufe sind als GV1 (spontane Geburt), GV2 (mäßige Zughilfe, teilweise mit Einsatz des mechanischen Geburtshelfers, 18 von 34 Fällen) und GV3 (starke Zughilfe durch Personen und mechanischen Geburtshelfer) bezeichnet worden. Kälber aus Zwillingsgeburten und aus Geburten durch Kaiserschnitt sind in die Untersuchungen nicht einbezogen worden.

Untersuchungsablauf, Messungen: Mit Hilfe der Tageszeiten von Geburt und Messung wurde die Zeit zwischen Geburt und Messung (ZGM) errechnet. Blutproben wurden zuerst beim Kalb, welches in Seitenlage durch eine Person fixiert war, durch leichte Stauung mit der Hand aus der V. jugularis entnommen. Eine Speichelprobe wurde mit Hilfe eines Tampons gewonnen ( etwa 30 bis $60 \mathrm{sec}$ in der Mundhöhle des Tieres) und in ein Zentrifugenglas aufgenommen. Die Messung der Körpertemperatur erfolgte mit gebräuchlichen Fieberthermometern. Die Kühe wurden mit einem Strickhalfter fixiert, dann wurde mit Hilfe einer Staukette und durch Punktion der V. jugularis eine Blutprobe gewonnen. Danach erfolgte die Messung der Körpertemperatur bei der Kuh. Die Blutproben wurden in Monovetten aufgenommen, in einem Kühlgefäß in das Labor des Institutes gebracht und dort unverzüglich bearbeitet. Das Plasma wurde eingefroren und zu einem späteren Zeitpunkt blockweise bearbeitet. Der Säure-Basen-Status und $\mathrm{Hb}$ wurden mit dem AVL 995-Hb Automatic Blood Gas System von Biomedical Instruments Graz, Österreich, Hk mit der Mikromethode und Cortisol mit einem RIA bestimmt. Aus Hb und Hk wurde MCHC errechnet.

Bearbeitung der Ergebnisse, statistische Methoden: Die Bearbeitung der Ergebnisse ist mit Systat von SPSS Science Software vorgenommen und die Varianzanalyse angewandt worden, wobei die Laktationsnummer (LANR) des Muttertieres, das Geschlecht des Kalbes, die Geburtsverlaufsgruppen und mit Hilfe des $\mathrm{pH}$-Wertes des venösen Blutes des Kalbes gebildete Gruppen (pHG1: pH>7,330; pHG2: pH 7,280 - 7,329; pHG3: pH 7,230 - 7,279 und pHG4: pH<7,229) als Kategorien genutzt wurden. Die LANR wurden zu LANR-Gruppen zusammengefasst (LAG1: LANR1; LAG2: LANR 
2; LAG3: LANR 3; LAG4: LANR 4 bis 9). Mit dem Mixed Regression Model wurden Effekte numerischer Variablen und von Kategorien auf Plasmacortisol des Kalbes und der Kuh geprüft. Quantil-Quantil Plots (Q-Q Plots) wurden genutzt, um unterschiedliche Verteilungen der Variablen innerhalb und zwischen Kategorien deutlich zu machen. Die Irrtumswahrscheinlichkeiten (Bonferroni adjusted probability) sind in den Tabellen und Abbildungen angegeben und, wenn nicht ausdrücklich erwähnt, mit 5 \% angenommen worden.

\section{Ergebnisse}

\section{Trächtigkeitsdauer der Kuh, Geschlecht und Körpermasse der Kälber}

Zwischen KM der Kälber und TD der Kuh ließ sich eine direkte Beziehung nachweisen $(r=0,498 ; p<0,000 ; N$ 80), die bei weiblichen Kälbern stärker $(r=0,548 ; p<$ $0,000 ; \mathrm{N} 37)$ als bei männlichen ( $r=0,426$; $\mathrm{p}=0,004 ; \mathrm{N} 43$ ) war. In der Gruppe GV2 und GV3 war die Anzahl männlicher Kälber doppelt so groß wie diejenige weiblicher Kälber (Tab. 1). Weibliche Kälber hatten eine kleinere KM als männliche (m 46,6 \pm 0,8; w 43,9 \pm 0,9; p = 0,024). Mittelwertunterschiede der Körpermasse zwischen den Gruppen und zwischen den Geschlechtern in LAG1 bis LAG4 und in GV1 bis GV3 sowie in pHG1 bis pHG4 waren nicht zu sichern (Tab. 5).

Tabelle 1

Häufigkeit der Geburtsverläufe 1 bis 3 männlicher und weiblicher Kälber bei Kühen mit unterschiedlicher Laktationsnummer, Anzahl (Frequency of birth courses type 1 to 3 of male and female calves in cows with different lactation number)

\begin{tabular}{lrr|l|l|l|l|l|l|l|l}
\hline Laktationsnummer & 1 & 2 & 3 & 4 & 5 & 6 & 9 & \\
\hline $\begin{array}{l}\text { Geburtsverlauf 1 Kälber männlich } \\
\text { (spontane Geburt) }\end{array}$ weiblich & 1 & 6 & 7 & 3 & 1 & 2 & 1 & 21 \\
$\begin{array}{l}\text { Geburtsverlauf 2 Kälber männlich } \\
\text { (leichte Geburtshilfe) }\end{array}$ weiblich & 1 & 9 & 10 & 2 & 1 & 3 & & 26 \\
$\begin{array}{l}\text { Geburtsverlauf 3 Kälber männlich } \\
\text { (starke Geburtshilfe) }\end{array} \quad$ weiblich & 6 & 10 & 4 & & & & & 20 \\
\hline & & 1 & 2 & 5 & 1 & & 1 & & 10 \\
\hline
\end{tabular}

\section{Blutmesswerte und Körpertemperatur der Kühe und der Kälber}

Kälber hatten signifikant kleinere RT ( $=0,027)$, Hb $(p<0,000)$, MCHC $(p<0,000)$ und signifikant größere Plasmacortisolkonzentrationen $(p<0,000)$ als Kühe. Der Mittelwert von Hk war zwischen Kuh und Kalb nicht signifikant verschieden. Zwischen RT der Kuh und RT des Kalbes ließ sich für die Gesamtheit eine Korrelation nachweisen ( $r=0,327 ; p=0,003 ; N$ 80). Mit Hilfe des Mixed Regression Models konnten signifikante Effekte auf die Plasmacortisolkzentration der Kälber durch ZGM, Geburtstyp, $\mathrm{pCO}_{2}$ und die pH-Wertgruppe und auf diejenige der Kühe durch ZGM und $\mathrm{pCO}_{2}$ des Blutes des Kalbes nachgewiesen werden.

\section{Gruppen nach der Laktationsnummer der Kuh}

In den Gruppen waren die Mittelwertunterschiede zwischen Kuh und Kalb für RT und Hk nicht, diejenigen von $\mathrm{Hb}$, MCHC und Plasmacortisol sicher. Die Beziehung zwischen RT der Kuh und des Kalbes war nur in LAG2 sicher $(r=0,468 ; p=0,014$; $N$ 27). Im Falle von Plasmacortisol war die Beziehung in LAG2 ( $\mathrm{r}=0,457 ; \mathrm{p}=0,016)$, in LAG3 $(r=0,411 ; p=0,033)$ und in LAG4 $(r=0,512$; $p=0,043)$ sicher. Für die Mittelwerte der Variablen konnten zwischen LAG1 bis LAG4 nur für RT sichere Unter- 
schiede zwischen LAG1 und LAG4 $\left(39,3^{\circ} \mathrm{C}\right.$ gegenüber $38,83{ }^{\circ} \mathrm{C}$; $\left.\mathrm{p}=0,028\right)$ nachgewiesen werden. Unterschiedliche Häufigkeiten der Geburtstypen in den Gruppen nach der LANR der Kuh (Tab. 1) waren nicht sicher.
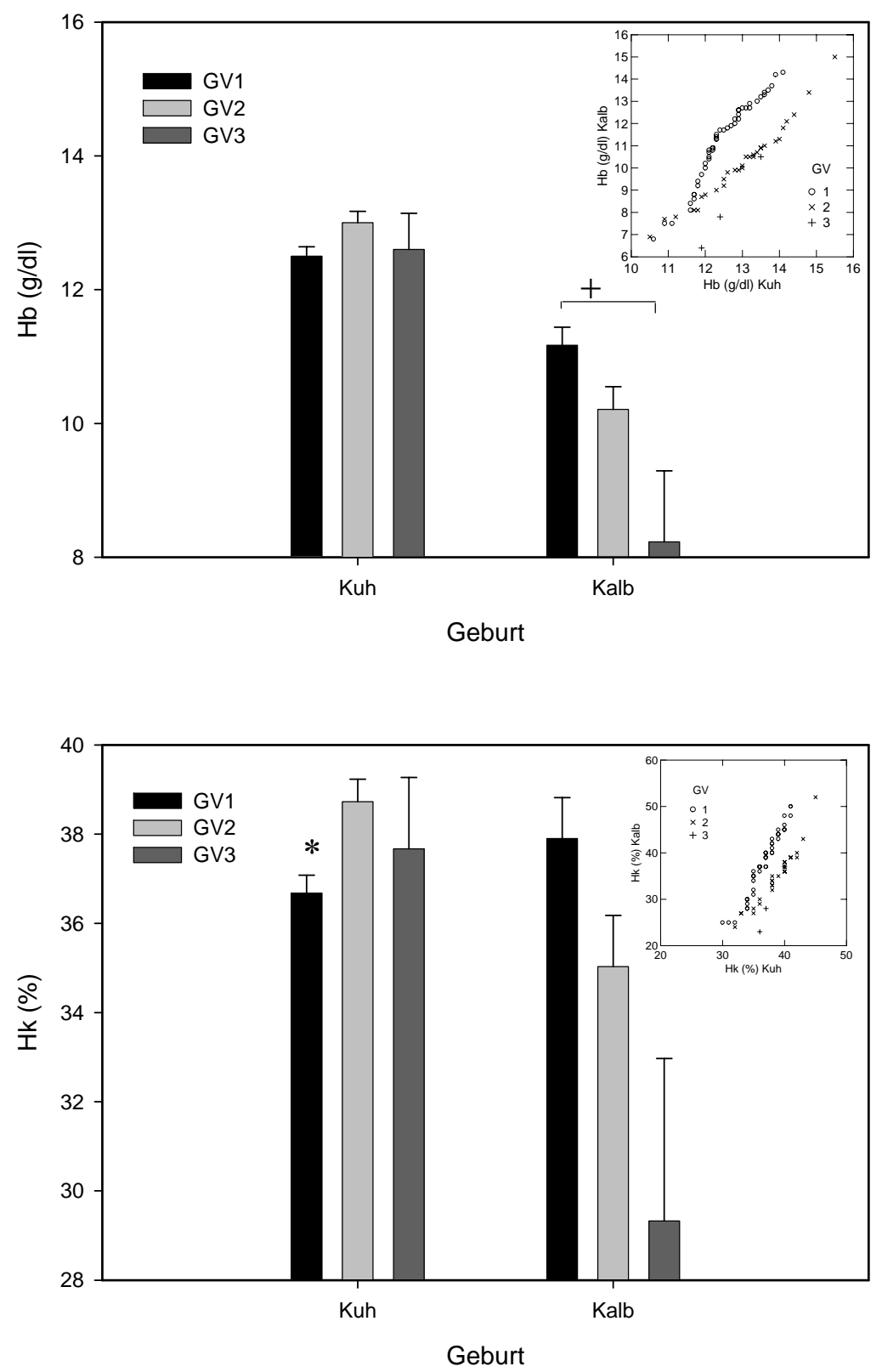

Abb. 1: Hämoglobinkonzentration (Hb, oberer Teil) und Hämatokrit (Hk, unterer Teil) bei Kühen und Kälbern nach der Geburt, Gruppen nach Geburtstyp, Least Squares Means und SEM, Insert: Q-Q Plots von Hb und Hk der Kuh und des Kalbes (Hemoglobin concentration (Hb, upper part) and hematocrite (Hk, lower part) in cows and calves after birth, groups by birth type, Least Squares Means and SEM, Insert: Quantile-Quantile Plots of Hb and $\mathrm{Hk}$ of the cow and the calf)

\section{Gruppen nach dem Geburtsverlauf}

In GV2 war für RT ( $=0,029)$ und Hk $(p=0,001)$ ein sicherer Mittelwertunterschied zwischen Kuh und Kalb nachzuweisen. Für MCHC und Plasmacortisol waren die Mittelwertunterschiede zwischen Kuh und Kalb in GV1 bis GV3 in gleicher Stärke vorhanden. Die Mittelwerte von $\mathrm{Hb}$ und $\mathrm{Hk}$ waren bei Kühen mit GV2 größer als bei jenen mit GV1 und GV3 (Abb. 1), bei Kälbern wurden Hb, Hk und MCHC von GV1 
nach GV3 kleiner (Abb. 1 und 2). Mittelwertunterschiede von $\mathrm{Hb}$ und Hk zwischen GV1 bis GV3 traten deutlicher hervor, wenn die Differenzen (Kalb-Kuh) der Variablen für die Bewertung herangezogen wurden (Abb. 3). Plasmacortisol wurde bei Kühen und Kälbern von GV1 nach GV3 größer, signifikant waren die Mittelwertunterschiede zwischen GV1 und GV2 sowie GV3 (Abb. 2). Direkte Beziehungen zwischen Plasmacortisol der Milchkuh und des neugeborenen Kalbes waren für die Gesamtheit sicher $(r=0,445 ; p<0,000 ; N$ 80), bei GV1 nicht $(r=0,252 ; p=0,087 ; N$ 47), bei GV2 jedoch signifikant ( $r=0,435 ; \mathrm{p}=0,016 ; \mathrm{N} 30)$. Die Hormonkonzentrationen des Kalbes hatten mit den Differenzen der Konzentrationen Kalb-Kuh in den GV-Gruppen engere Beziehungen als jene der Kuh (Tab. 2). Die Q-Q Plots der Variablen bei Milchkühen und bei deren Kälbern (Insert bei Abb. 1 und 2) ließen die Qualität der Häufigkeitsverteilungen und die Unterschiede zwischen den Geburtsverlaufsformen deutlich erkennen. Zwischen Plasmacortisol und Speichelcortisol ließ sich für die Gesamtheit eine Korrelation nachweisen ( $r=0,565 ; \mathrm{p}<0,000 ; \mathrm{N} 66)$. Diese war in GV1 stärker ( $r=0,638 ; \mathrm{p}<0,000 ; \mathrm{N} 36)$ als in GV2 ( $\mathrm{r}=0,415 ; \mathrm{p}=0,028 ; \mathrm{N} 28)$. Plasmacortisol der Kuh und des Kalbes wiesen zur Tageszeit der Geburt Beziehungen auf, die durch polynomiale Regressionen zu charakterisieren waren (Kuh: $y=-0,23 x^{2}+$ $5,396 x+16,57, R^{2}=0,311$; Kalb: $\left.y=-1,56 x^{2}+40,42 x+54,47, R^{2}=0,225\right)$. An den Q-Q Plots von Tageszeit und Plasmacortisol sowie Tageszeit und Speichelcortisol sind die Unterschiede zwischen GV1, GV2 und GV3 und die Abweichungen von der Normalverteilung zu sehen (Abb. 5).

Tabelle 2

Regressionsgleichungen der Differenzen der Variablen Kalb-Kuh mit den Variablen der Kuh und des neugeborenen Kalbes und Bestimmtheitsmaß $\left(\mathrm{R}^{2}\right)$ sowie der kalkulierte X-Wert im Falle $\mathrm{Y}=0$ (Regressions of the differences of the variables calf-cow with the variables of the cow and that of the newborn calf and coefficient of determination $\left(R^{2}\right)$ as well as the calculated $x$-value in the case $y=0$ )

\begin{tabular}{|c|c|c|c|c|c|}
\hline & $\begin{array}{l}\text { RT } \\
\text { Kalb-Kuh } \\
\left({ }^{\circ} \mathrm{C}\right) \\
\end{array}$ & $\begin{array}{l}\text { Hb } \\
\text { Kalb-Kuh } \\
(\mathrm{g} / \mathrm{dl})\end{array}$ & $\begin{array}{l}\mathrm{Hk} \\
\text { Kalb-Kuh } \\
(\%) \\
\end{array}$ & $\begin{array}{l}\text { MCHC } \\
\text { Kalb-Kuh } \\
(\%) \\
\end{array}$ & $\begin{array}{l}\text { P-Cortisol } \\
\text { Kalb-Kuh } \\
(\mathrm{nmol} / \mathrm{l})\end{array}$ \\
\hline Kuh & $\begin{array}{l}y=-0,68 x+26,4 \\
R^{2}=0,349 \\
x=38,83\end{array}$ & $\begin{array}{l}y=-0,996 x+ \\
10,65 \\
R^{2}=0,196 \\
x=10,69\end{array}$ & $\begin{array}{l}y=-0,639 x+ \\
23,03 \\
R^{2}=0,091 \\
x=36,05\end{array}$ & $\begin{array}{l}\mathrm{y}=-0,906 \mathrm{x}+ \\
26,11 \\
\mathrm{R}^{2}=0,491 \\
\mathrm{x}=28,83\end{array}$ & $\begin{array}{l}y=2,24 x+122 \\
R^{2}=0,106 \\
x=54,55\end{array}$ \\
\hline Kalb & $\begin{array}{l}y=0,67 x-26,1 \\
R^{2}=0,324 \\
x=39,09\end{array}$ & $\begin{array}{l}y=0,999 x- \\
12,66 \\
R^{2}=0,802 \\
x=12,67\end{array}$ & $\begin{array}{l}y=0,91 x- \\
34,23 \\
R^{2}=0,784 \\
x=37,44\end{array}$ & $\begin{array}{l}y=0,89 x-30,6 \\
R^{2}=0,407 \\
x=34,39\end{array}$ & $\begin{array}{l}y=0,94 x- \\
19,95 \\
R^{2}=0,983 \\
x=21,25\end{array}$ \\
\hline
\end{tabular}

\section{Gruppen nach dem pH-Wert des Blutes der Kälber}

Unterschiedliche Häufigkeiten der Geburtstypen in den Gruppen waren schwach signifikant ( $\mathrm{p}=0,018)$. Wegen der spärlichen Besetzung ( $<5$ Fälle) einiger Felder ist dieses Ergebnis vorsichtig zu bewerten (Tab. 3). Negative Beziehungen zwischen pHWert und Plasmacortisol ( $\mathrm{r}=-0,308 ; \mathrm{p}=0,005 ; \mathrm{N} 80$ ) sowie $\mathrm{pH}-$ Wert und Speichelcortisol ( $\mathrm{r}=-0,424 ; \mathrm{p}<0,000 ; \mathrm{N}$ 66) und positive Beziehungen zwischen $\mathrm{pCO}_{2}$ und Plasmacortisol $\left(\mathrm{r}=0,452 ; \mathrm{p}<0,000 ; \mathrm{N}\right.$ 80) sowie $\mathrm{pCO}_{2}$ und Speichelcortisol $(\mathrm{r}=$ 0,428; $\mathrm{p}<0,000$; N 66) bei Kälbern waren sicher. Die Mittelwerte der Plasmacortisolkonzentration unterschieden sich zwischen pHG1 bis pHG4 bei den Kühen nicht, bei den Kälbern, wie auch diejenigen von $\mathrm{ZGM}$, $\mathrm{pCO}_{2}, \mathrm{BE}$ und $\mathrm{HCO}_{3}$ und Plasmacortisol signifikant (Tab. 4). Korrelationen zwischen Plasmacortisol und Speichelcortisol waren in den Gruppen signifikant $(r=0,501$ bis $r=0,76)$. 

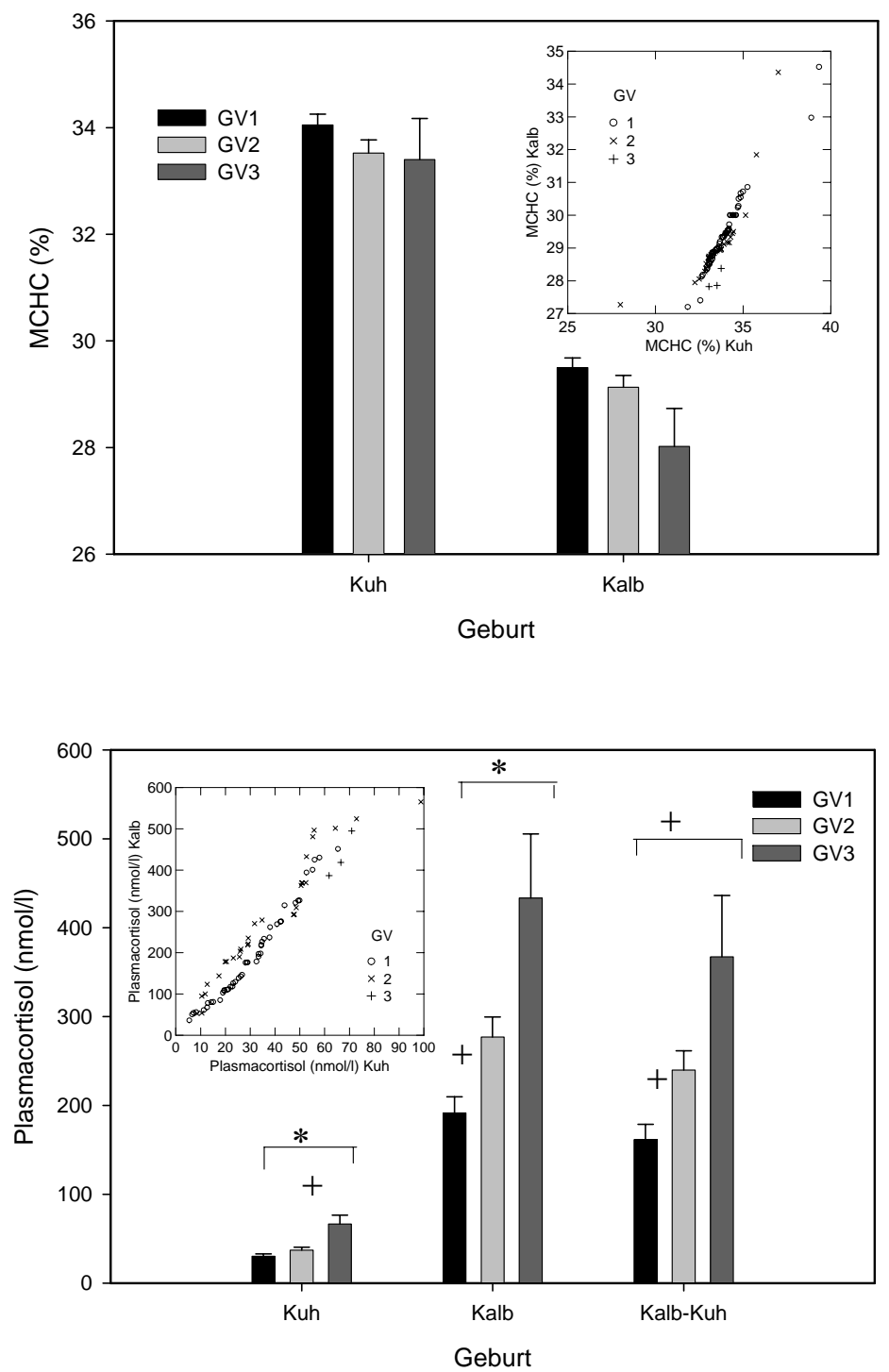

Abb. 2: Mittlere korpuskuläre Hämoglobinkonzentration (MCHC, oberer Teil) und Plasmacortisolkonzentration (unterer Teil) bei Kühen und Kälbern nach der Geburt sowie die Differenz Plasmacortisol Kalb-Kuh, Gruppen nach Geburtstyp, Least Squares Means und SEM, Insert: Q-Q Plots von MCHC und Plasmacortisol der Kuh und des Kalbes (Mean Corpuscular Hemoglobin Concentration (MCHC, upper part) and plasma cortisol concentration (lower part) in cows and calves after birth as well as the difference of plasma cortisol calf-cow, groups by type of birth, Least Squares Means and SEM, Insert: Quantile-Quantile Plots of MCHC and plasma cortisol of the cow and the calf)

Tabelle 3

Häufigkeit der Geburtsverläufe 1 bis 3 männlicher und weiblicher Kälber in den Gruppen nach dem pH-Wert des venösen Blutes des Kalbes (siehe Material und Methoden), Anzahl (Frequency of the birth courses type 1 to 3 of male and female calves in groups by $\mathrm{pH}$-value of calf venous blood (see material and methods))

\begin{tabular}{|c|c|c|c|c|c|}
\hline & pHG1 & pHG2 & pHG3 & pHG4 & \\
\hline $\begin{array}{lr}\begin{array}{l}\text { Geburtsverlauf } 1 \\
\text { (spontane Geburt) }\end{array} & \text { Kälber männlich } \\
\text { Geburtsverlauf } 2 & \text { Kälber männlich } \\
\text { (leichte Geburtshilfe) } & \text { weiblich } \\
\begin{array}{l}\text { Geburtsverlauf } 3 \\
\text { (starke Geburtshilfe) }\end{array} & \text { Kälber männlich } \\
\end{array}$ & $\begin{array}{l}6 \\
7 \\
3 \\
3\end{array}$ & $\begin{array}{l}11 \\
9 \\
3 \\
1\end{array}$ & $\begin{array}{l}1 \\
6 \\
8 \\
2 \\
1\end{array}$ & $\begin{array}{l}3 \\
4 \\
6 \\
4 \\
1 \\
1\end{array}$ & $\begin{array}{l}21 \\
26 \\
20 \\
10 \\
2 \\
1\end{array}$ \\
\hline & 19 & 24 & 18 & 19 & 80 \\
\hline
\end{tabular}




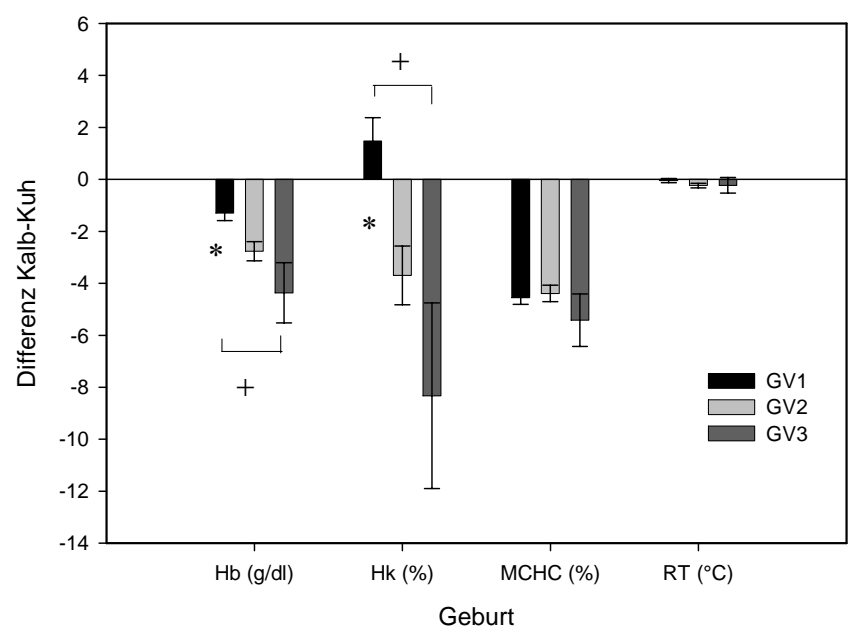

Abb. 3: Differenzen von Hb, Hk, MCHC und RT zwischen Kalb und Kuh nach der Geburt, Gruppen nach Geburtstyp, Mittelwerte und Standardabweichung (Differences of Hb, Hk, MCHC and RT between calf and dam after birth, groups by type of birth, Mean Values and Standard Deviation)

Tabelle 4

Kohlendioxidpartialdruck $\left(\mathrm{pCO}_{2}\right)$, Basenüberschuss (BE) und Standardbikarbonat $\left(\mathrm{HCO}_{3}\right)$ sowie Plasma- und Speichelcortisol und Zeit Geburt-Messung der Gruppen nach dem pH-Wert des venösen Blutes des Kalbes, Least Squares Means und SEM, Anzahl der Tiere in Klammern (Carbon dioxid pressure, base excess and standard bicarbonate as well as plasma and saliva cortisol and time between birth and measurement in groups by $\mathrm{pH}$-value of venous blood of the calf, Least Squares Means and SEM, number of animals in parentheses)

\begin{tabular}{|c|c|c|c|c|}
\hline & pH Gruppe 1 & pH Gruppe 2 & pH Gruppe 3 & pH Gruppe 4 \\
\hline \multirow{3}{*}{$\begin{array}{l}\mathrm{pCO}_{2} \\
(\mathrm{mmHg})\end{array}$} & (19) & (24) & (18) & (19) \\
\hline & $55,2^{\mathrm{a}}$ & $60,04^{\mathrm{a}}$ & $65,6^{\mathrm{b}}$ & $73,0^{b, c}$ \\
\hline & 1,5 & 1,3 & 1,5 & 1,5 \\
\hline \multirow{2}{*}{$\begin{array}{l}\mathrm{BE} \\
(\mathrm{mmol} / \mathrm{l})\end{array}$} & $5,65^{\mathrm{a}}$ & $3,08^{\mathrm{a}, \mathrm{c}}$ & $1,18^{\mathrm{b}, \mathrm{c}}$ & $-4,56^{\mathrm{b}, \mathrm{d}}$ \\
\hline & 0,75 & 0,67 & 0,78 & 0,75 \\
\hline \multirow{2}{*}{$\begin{array}{l}\mathrm{HCO}_{3} \\
(\mathrm{mmol} / \mathrm{l})\end{array}$} & $30,06^{\mathrm{a}}$ & $28,54^{\mathrm{a}}$ & $27,59^{\mathrm{a}}$ & $23,6^{\mathrm{b}}$ \\
\hline & 0,65 & 0,58 & 0,67 & 0,65 \\
\hline \multirow{2}{*}{$\begin{array}{l}\text { Plasmacortisol Kuh } \\
\text { (nmol/l) }\end{array}$} & 26,0 & 32,3 & 41,4 & 38,0 \\
\hline & 4,16 & 3,7 & 4,3 & 4,2 \\
\hline \multirow{2}{*}{$\begin{array}{l}\text { Plasmacortisol Kalb } \\
(\mathrm{nmol} / \mathrm{l})\end{array}$} & $170,1^{\mathrm{a}}$ & $194,0^{c}$ & $288,5^{b, c}$ & $291,0^{\mathrm{b}, \mathrm{c}}$ \\
\hline & 29,1 & 25,9 & 29,9 & 29,1 \\
\hline Plasmacortisoldifferenz & $144,1^{\mathrm{a}}$ & $161,8^{\mathrm{c}}$ & $247,1^{\mathrm{c}}$ & $253,0^{\mathrm{b}, \mathrm{c}}$ \\
\hline \multirow[t]{2}{*}{ Kalb-Kuh (nmol/l) } & 27,8 & 24,8 & 28,6 & 27,8 \\
\hline & (13) & $(22)$ & (12) & (19) \\
\hline \multirow{3}{*}{$\begin{array}{l}\text { Speichelcortisol Kalb } \\
(\mathrm{nmol} / \mathrm{l})\end{array}$} & 7,8 & 9,3 & 17,9 & 16,6 \\
\hline & 3,2 & 2,4 & 3,3 & 2,6 \\
\hline & (19) & (24) & (18) & (19) \\
\hline \multirow{2}{*}{$\begin{array}{l}\text { Zeit Geburt-Messung } \\
\text { (min) }\end{array}$} & $561^{a}$ & $390^{c}$ & $277 \mathrm{~b},{ }^{\mathrm{c}}$ & $196^{\mathrm{b}, \mathrm{c}}$ \\
\hline & 59 & 53 & 61 & 59 \\
\hline
\end{tabular}



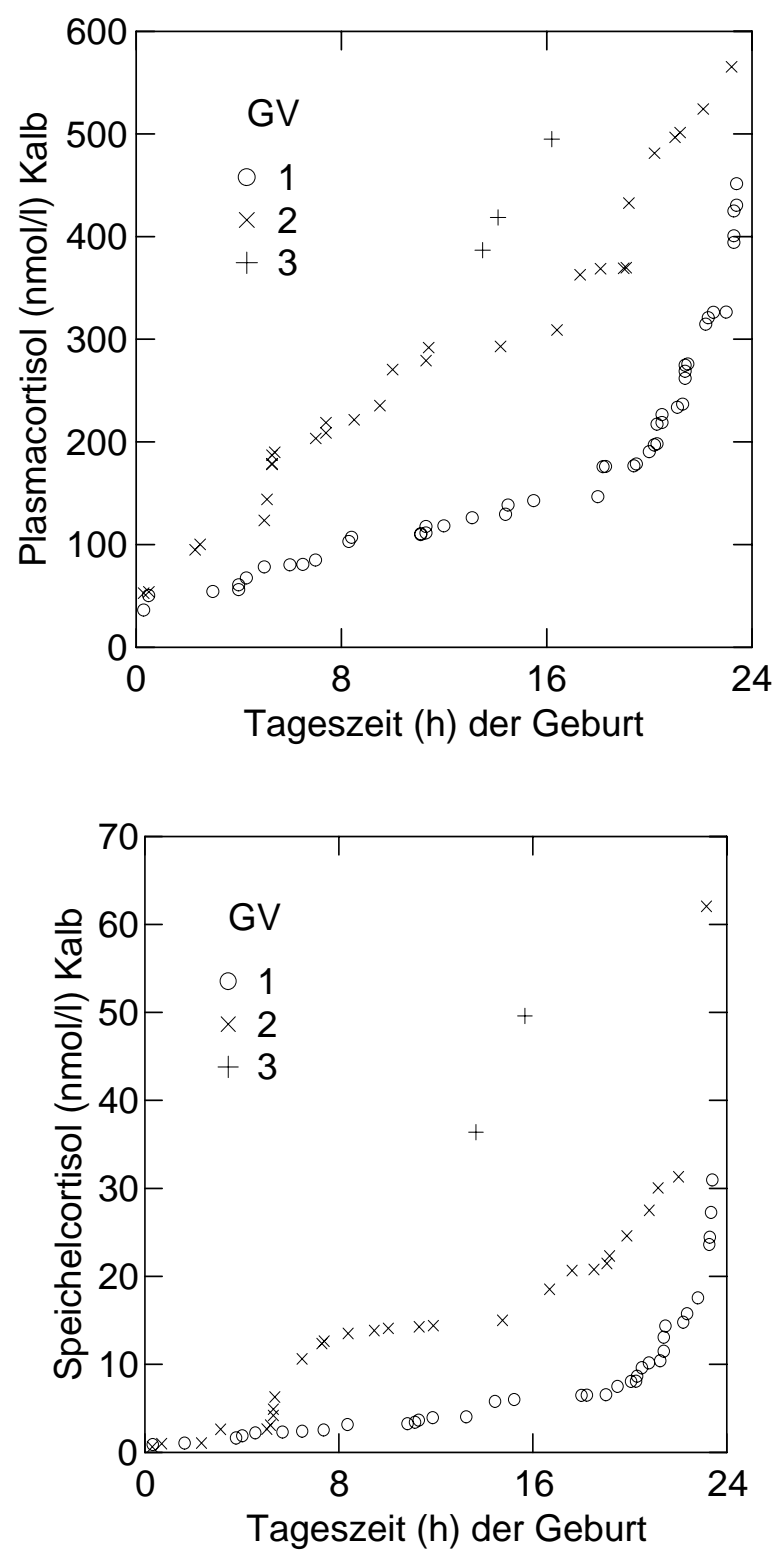

Abb. 4: Q-Q Plots der Tageszeit der Geburt und der Plasmacortisolkonzentration des Kalbes (oberer Teil) sowie der Tageszeit der Geburt und der Speichelcortisolkonzentration des Kalbes (unterer Teil) (Quantile-Quantile Plots of day time of birth and plasma cortisol concentration of the calf (upper part) as well as of day time of birth and the saliva cortisol concentration of the calf (lower part))

4.

Diskussion

Durch die Untersuchungen wurde ein Einfluss der vom Betreuungspersonal vorgenommenen geburtshilflichen Maßnahmen auf die Variation physiologischer Messwerte der Kälber in gebräuchlichen betrieblichen Bedingungen der Milchrindhaltung aufgezeigt. Effekte der untersuchten Faktoren auf die Körpertemperatur waren bei Kühen geringgradig und nur in weniger Fällen sicher. Die Mechanismen, die die postpartale Steigerung der Wärmebildung gewährleisten, schienen bei den untersuchten Kälbern nicht eingeschränkt gewesen zu sein, denn extrem niedrige Körpertemperaturen wie bei Holstein Friesian Kälbern aus sehr schwierigen Geburten (VERMOREL et al., 1989) konnten nicht festgestellt werden. Unterschiedliche Hormonkonzentrationen bei Tiergruppen mit verschiedenen Geburtsverläufen (Abb. 2 und 
Tabelle 5

Körpermassen männlicher (m) und weiblicher (w) Kälber in den Gruppen nach der Laktationsnummer der Kuh (LAG1 bis LAG4), nach dem Geburtsverlauf (GV1 bis GV3) und nach dem pH-Wert des Blutes des Kalbes (pHG1 bis pHG4), Least Squares Means and SEM, ( Body weight of male (m) and female (w) calves, groups by the lactation number of the dam (LAG1 to LAG4), by the type of birth (GV1 to GV3) and by the pH value of calf blood (pHG1 to pHG4), Least Squares Means and SEM

\begin{tabular}{|c|c|c|c|c|c|c|c|c|}
\hline & \multicolumn{2}{|l|}{ LAG1 } & \multicolumn{2}{|l|}{ LAG2 } & \multicolumn{2}{|l|}{ LAG3 } & \multicolumn{2}{|l|}{ LAG4 } \\
\hline $\begin{array}{l}\text { KM (kg) } \\
\text { KM (kg) }\end{array}$ & $\begin{array}{c}45,7 \\
1,7 \\
\mathrm{~m}(7) \\
47,7 \\
1,97\end{array}$ & $\begin{array}{l}\mathrm{w}(3) \\
41 \\
3\end{array}$ & $\begin{array}{l}44,4 \\
1,03 \\
\mathrm{~m}(16) \\
46,3 \\
1,3\end{array}$ & $\begin{array}{l}\text { w }(11) \\
41,6 \\
1,57\end{array}$ & $\begin{array}{l}45,9 \\
1,03 \\
\mathrm{~m}(12) \\
46,5 \\
1,5\end{array}$ & $\begin{array}{l}\text { w (15) } \\
45,3 \\
1,34\end{array}$ & $\begin{array}{l}45,9 \\
1,34 \\
\mathrm{~m}(8) \\
46,38 \\
1,84\end{array}$ & $\begin{array}{l}\mathrm{W}(8) \\
45,5 \\
1,84\end{array}$ \\
\hline & \multicolumn{2}{|l|}{ GV1 } & \multicolumn{2}{|l|}{ GV2 } & \multicolumn{2}{|l|}{ GV3 } & & \\
\hline KM (kg) & \begin{tabular}{|l|}
44,3 \\
0,76 \\
$\mathrm{~m}(21)$ \\
45,9 \\
1,1
\end{tabular} & $\begin{array}{l}\text { W (26) } \\
43,1 \\
1\end{array}$ & $\begin{array}{l}46,7 \\
0,96 \\
\mathrm{~m}(20) \\
46,8 \\
1,1\end{array}$ & $\begin{array}{l}\text { w 10) } \\
46,7 \\
1,6\end{array}$ & $\begin{array}{l}47,33 \\
3,02 \\
\mathrm{~m}(2) \\
52,5 \\
3,6\end{array}$ & $\begin{array}{l}\mathrm{w}(1) \\
37\end{array}$ & & \\
\hline KM (kg) & \multicolumn{2}{|l|}{ pHG1 } & \multicolumn{2}{|l|}{ pHG2 } & \multicolumn{2}{|l|}{ pHG3 } & \multicolumn{2}{|l|}{ pHG4 } \\
\hline KM (kg) & $\begin{array}{l}46,95 \\
1,2 \\
\mathrm{~m}(9) \\
47 \\
1,65 \\
\end{array}$ & $\begin{array}{l}\mathrm{w}(10) \\
46,9 \\
1,56\end{array}$ & $\begin{array}{l}43,71 \\
1,07 \\
\mathrm{~m}(14) \\
45,43 \\
1,32\end{array}$ & $\begin{array}{l}\text { w }(10) \\
41,3 \\
1,56\end{array}$ & $\begin{array}{l}46,44 \\
1,24 \\
\mathrm{~m}(10) \\
46,3 \\
1,56\end{array}$ & $\begin{array}{l}\text { w (8) } \\
46,63 \\
1,75\end{array}$ & $\begin{array}{l}44,79 \\
1,2 \\
\mathrm{~m}(10) \\
48,1 \\
1,56\end{array}$ & $\begin{array}{l}\mathrm{w}(9) \\
41,11 \\
1,65\end{array}$ \\
\hline
\end{tabular}

4) lassen, wie auch verzögerte Abfälle derselben nach der Geburt sowie in den folgenden Tagen (HOYER et al. 1990; STOTT und REINHARD, 1978), die Reaktivität der Tiere in Abhängigkeit von der Vorgehensweise bei der Geburtshilfe und bei den Messungen und auch von den Umgebungsbedingungen erkennen. Größere Cortisolwerte der Färsen nach der Kalbung (GRÜNBERG, 1996; STEINHARDT et al., 1994a) als bei multiparen Kühen und eine Häufung der assistierten Geburten bei Kühen mit LANR 1 bis 3 (Tab. 1) deuten auf Einflüsse der Herdenstruktur hin, die hier nicht gesichert werden konnten. Größere Hb und Hk der Kühe mit GV2, die Ausdruck sympathischer Aktivierung und/oder relativ kleiner Plasmavolumen sind (Abb. 1), sprechen, wie auch signifikant höhere Cortisolwerte (71,8 \pm 58,6 ng/ml) junger Mutterkühe mit männlichen Kälbern nach der Kalbung gegenüber jenen mit weiblichen Kälbern (43,2 \pm 42,5 ng/ml) (GRÜNBERG, 1996) dafür, dass gut entwickelte Nachkommen mit absolut oder relativ großer Körpermasse, häufiger männliche Kälber als weibliche (Tab. 1), stärkere Geburtsbelastungen verursachen und Anlaß für geburtshilfliche Eingriffe geben können. BELLOWS und LAMMOGLIA (2000) fanden bei primiparen Kreuzungstieren (Hereford, Limousin, Piedmontese) hochsignifikante Unterschiede der mittleren Geburtsgewichte der Kälber, aber keine sicheren Unterschiede der innerhalb von 30 min post partum ermittelten Serumcortisolkonzentrationen zwischen 4 Geburtstypen. Bei jungen Mutterkühen (Deutsche Schwarzbunte im alten Typ und Holstein Friesian; Gruppenabkalbungen außerhalb des Stalles) und bei deren Kälbern waren Unterschiede der Cortisolwerte zwischen 4 Geburtstypen vorhanden, jedoch wegen der großen Streuung nicht zu sichern (GRÜNBERG, 1996).

Positive Beziehungen zwischen Plasmacortisol von Kuh und Kalb deuten darauf hin, dass gleichgerichtete Änderungen vorkommen (HYDBRING et al., 1999), und dass 
ein diaplacentärer Austausch von Cortisol eine Rolle spielen könnte. Signifikante Effekte von $\mathrm{pCO}_{2}$ sowie der $\mathrm{pH}$-Wertgruppen auf Plasmacortisol der Kälber (Tab. 4), die auch von MASSIP (1980a,b) bei der Untersuchung klinisch relevanter Tiergruppen angesprochen wurden und nicht sicher waren, deuten zunächst auf die gleichzeitige Änderung dieser Variabeln hin. Kleinere Hb und Hk der Kälber mit GV2 und GV3, die zum Teil durch gestörte placento-fetale Transfusion während des Geburtsverlaufes bedingt sind (STEINHARDT et al., 1994b, 1995, 1996), schränken die Anpassungskapazität der Kälber ein, wie es im Falle der pH-Wertgruppe 3 offensichtlich ist. In dieser Gruppe waren auch Mittelwertunterschiede zwischen Kuh und Kalb für RT ( $\mathrm{p}=$ 0,049) und für Hk ( $\mathrm{p}=0,006)$ sicher, während diejenigen für Hb, MCHC und Plasmacortisol in allen $\mathrm{pH}$-Wertgruppen sicher waren. Die Korrelation zwischen $\mathrm{pCO}_{2}$ und Plasmacortisol war in dieser Gruppe eng ( $r=0,642 ; p=0,004 ; \mathrm{N} 18$ ).

Eine Anpassung der Sauerstofftransportkapazität und speziell von $\mathrm{Hb}$ (RICHARDSON und BOCKING, 1998) sowie der Sensitivität der Nebennierenrinde in Abhängigkeit von der Sauerstoffverfügbarkeit während des letzten Drittels des intrauterinen Lebens (DUCSAY, 1998; MUROTSUKI et al., 1996) geben hinsichtlich der Analyse der Ursache-Wirkung-Beziehung Anlass zu der Frage, in welchem Maße die Geburtsbedingungen und die geburtshilflichen Maßnahmen und/oder die Lebensbedingungen vor der Abkalbung bzw. Geburt zu der unterschiedlichen Entwicklungsqualität (STEINHARDT et al., 1992, 1993) und Reaktionsfähigkeit der Nachkommen und auch der Muttertiere (BRAASTAD et al., 1998; LAY et al., 1997) beigetragen haben können. Die fetale Nebenniere ist sensitiver gegenüber pathophysiologischen Erscheinungen der Gravidität und der Geburtsphase wie geändertem placentalem Blutgasaustausch und Reduzierung der placentären metabolischen Kapazität als allein gegenüber dem Säure-Basen-Status des Fetus (HARLIN et al., 1993; ROSSDALE et al., 1995), der gegen Ende der Gravidität größere Variationen aufweisen kann (WOUDSTRA et al., 1995). Eine starke peripartale Stimulation durch Trophormone und eine unterschiedliche Reaktion der Nebenniere in Abhängigkeit von der Entwicklungsqualität der Nachkommen und deren Geburtsbedingungen geht aus mehreren Untersuchungen hervor (PARKER, 1996; ROSSDALE et al., 1995). Beziehungen unterschiedlichen Grades zwischen den Differenzen der Variablen KalbKuh zu den Variablen der Kuh und des Kalbes (Tab. 2) sind ein Ausdruck der individuellen Anpassungsgrade an spezielle Umgebungsbedingungen. Die errechneten XWerte im Falle $\mathrm{y}=0$ weisen auf den Bereich von bekannten Referenzwerten hin. Diese Anpassung hat zur Folge, dass die individuellen Änderungen physiologischer Variablen der Kühe und der Kälber nach der Geburt von unterschiedlichen Niveaus aus erfolgen und in unterschiedlichem Grade vonstatten gehen (GRÜNBERG, 1996; STEINHARDT et al. 1995; STEINHARDT und THIELSCHER, 2000).

Poolgröße, Verteilungsvolumen und Halbwertzeit des Cortisols sind von der Menge des CBG (BERDUSCO et al., 1994; KATTESH et al., 1997; SERALINI, 1996) sowie des Albumin und von den chemisch-physikalischen Eigenschaften des Mediums abhängig. Die Gesamtmenge des intravasalen Proteins und die Anteile der Proteinqualitäten (Fetuin, Albumin und auch CGB) ändern sich bei Kälbern peripartal beträchtlich. Freies Cortisol im Blutplasma verlässt den extrazellulären Flüssigkeitsraum über Nieren und Drüsen wie Speicheldrüse (FRANCIS et al., 1987) und Milchdrüse (VERKERK et al., 1998). Die prinzipiell gleichen Änderungen der Konzentrationen des Cortisols werden durch positive Beziehungen zwischen Speichelcortisol und 
Plasmacortisol angezeigt. Ein stärkerer Einfluß weiterer Faktoren insbesondere auf die Disposition des Cortisols und auf dessen Übergang in Drüsengewebe und -sekrete ist an den schwächeren Korrelationen der Konzentrationen bei einigen Kategorien der Kälber zu erkennen. Circadiane und ultradiane Rhythmizität der Konzentrationen von ACTH und Cortisol im Blut von Schafen (SIMONETTA et al., 1991) und die Synchronisierung des Fetus infolge der placentären Passierbarkeit des Melatonin und der gleichartigen rhythmischen Variationen der Melatoninkonzentration im Blut des Muttertieres und des Fetus (McMILLEN et al., 1995) lassen eine von der Tageszeit der Geburt abhängige Reaktivität des HHN oder dadurch beeinflusste Disposition des Cortisols, die sich sowohl beim Kalb als auch bei der Kuh andeutete (Abb. 5), sehr wahrscheinlich erscheinen. Zu den durch die diurnale Rhythmizität bedingten hormonellen Effekten wären weitere Untersuchungen notwendig.

Die Aktivierung der Hormonproduktion des Kalbes während der Geburt ist abhängig von der Integrität des Hypothalamo-Hypophysen-Nebennierensystems (HHN). Eine forcierte Extraktion kann infolge stärkerer Druckwirkungen auf den Kopf und Halsbereich der Kälber zu einer Reizung und auch zu vorübergehender Einschränkung peripherer Komponenten regulatorischer Mechanismen (Rezeptoren, endokrine Drüsen) und zentralnervaler Funktionen beigetragen haben. Die Reaktivität des HHN, die Cortisoldisposition und auch die von der Hämoglobinmenge und -qualität abhängige Sauerstofftransportkapazität des Blutes sind von Bedeutung für die frühe postnatale Organentwicklung und Adaptationsfähigkeit der Kälber und auch für gezielte Supplementierungen in Verbindung mit der Nahrungsverfügbarkeit der Tiere. Zusammenhänge dieser Erscheinungen mit der Frequenz von direkten und indirekten Verlusten in der Kälberaufzucht wie auch die Anwendung geeigneter supplementierter Futtermittel wären in weiteren Untersuchungen zu prüfen.

\section{Literatur}

BELLOWS, R.A.; LAMMOGLIA, M.A.:

Effects of severity of dystocia on cold tolerance and serum concentrations of glucose and cortisol in neonatal beef calves. Theriogenology 53 (2000), 803-813

BERDUSCO, E.T.;. MILNE, W.K; CHALLIS, J.R.G.:

Low-dose cortisol infusion increases plasma corticosteroid-binding globulin (CBG) and the amount of hepatic CBG mRNA in fetal sheep on day 100 of gestation. J. Endocrinol. 140 (1994), 425-430

BRAASTAD, B.O.:

Effects of prenatal stress on behaviour of offspring of laboratory and farmed mammals. Appl. Anim. Behaviour Sci. 61 (1998), 159-180

DUCSAY, C.:

Fetal and meternal adaptations to chronic hypoxia: prevention of premature labor in response to chronic stress. Comp. Biochem. Physiol. 119A (1998), 675-681

EBERHART, R.J.; Patt, J.A.:

Plasma cortisol concentrations in newborn calves. Am J. Vet. Res. 32 (1971) 12,1921-1927

FRANCIS, S.J.; WALKER, R.F.; RIAD-FAHMY, D.; HUGHES, D.; MURPHEY, J.F.; GRAY, O. P.:

Assessment of adrenocortical activity in term newborn infants using salivary cortisol determinations. J. Pediatr. 111 (1987), 129-133

GRÜNBERG, W.:

Untersuchungen zur Eignung der Rinderrasse Deutsch Schwarzbunt (DSB) für eine ganzjährige Außenhaltung in besonderer Berücksichtigung der Abkalbung. Hannover, Vet. Med. Diss, 1996

HARLIN, C.A.; TUCKER, J.M.; WINKLER, C.L.; HENSON, B.; PARKER, C.R.:

Altered adrenal steroid production in term infants having respiratory acidosis. Acta Endocrinologica 128 (1993), 136-139

HEUWIESER, W.; HARTIG, U.; OFFENEY, F.; GRUNERT, E.:

Zur Bedeutung von Glukokortikoiden als Stressparameter beim Rind im peripartalen Zeitraum. J. Vet. Med. A 33 (1987), 178-187 
HOYER, C.; GRUNERT, E.; JÖCHLE, W.:

Plasma glucocorticoid concentrations in calves as an indicator of stress during parturition. Am J. Vet. Res. 51 (1990) 11, 1882-1884

HUNTER, J.T.; FAIRCLOUGH, R.J.; PETERSON, A.J.; WELCH, R.A.S.: Foetal and maternal hormonal changes preceding normal bovine parturition. Acta Endocrinologica 84 (1977), 653-662

HYDBRING, E.; MADEJ, A.; MacDONALD, E.; DRUGGE-BOHOLM, G.; BERGLUND, B.; OLSSON, K.: Hormonal changes during parturition in heifers and goats are related to the phases and severity of labour. J. Endocrinol. 160 (1999), 75-85

KATTESH, H.G.; BAUMBACH, G.A.; GILLESPIE, B.B.; SCHNEIDER, J.F.; MURAI, J.T.: Distribution between protein-bound and free forms of plasma cortisol in the gilt and fetal pig near term. Biol. Neonate 72 (1997), 192-200

KÜHNE, S.:

Untersuchungen über den Hämatokritwert und den Hämoglobingehalt des Blutes sowie den Gehalt des Blutplasmas an Gesamtprotein, freien Fettsäuren, Glukose, Laktat, Ca, Mg, Na, K, Pa, Fe, Gesamteisenbindungskapazität, Cu, und Zn bei Rindern und deren Kälbern vor und nach Aufnahme von Kolostrum. KMU Leipzig, Vet. Med. Diss., 1986

LAY, D.C.; RANDEL, R.D.; FRIEND, T.H.; CARROL, J.A.; WELSH, T.H.; JENKINS, O.C.; NEUENDORFF, D.A.; BUSHONG, D.M.; KAPP, G.M.:

MASSIP, A.: Effects of prenatal stress on the fetal calf. Domestic Animal Endocrinol. 14 (1997) 2, 73-80

The relation between the type of delivery and the acid-base and plasma cortisol levels of the newborn calf. Br. vet. J. 136 (1980a), 488-491

MASSIP, A.:

Relationship between $\mathrm{pH}$, plasma cortisol and glucose concentrations in the calf at birth. Br. vet. J. 136 (1980b), 597-601

McMILLEN, I.C.; HOUGHTON, D.C.; YOUNG, I.R.:

Melatonin and the development of circadian and seasonal rhythmicity. J. Reprod. Fertil, Suppl. 49 (1995), 137-146

MUROTSUKI, J. ; GAGNON, R. ; MATHEWS, S.G. ; CHALLIS, J.R.G.:

Effects of long-term hypoxemia on pituitary-adrenal function in fetal sheep. Am. J. Physiol. 271 (1996), E678-E685

PARKER, C.R.:

Developmental considerations. The fetal and neonatal endocrine response to stress. Contempary Endocrinology: Endocrinology of Critical Desease. Ed. K. P. OBER, Humana Press Inc., Totowa, NJ, 1996

RICHARDSON, B.S.; BOCKING, A.D.:

Metabolic and circulatory adaptations to chronic hypoxia in the fetus. Comp. Biochem. Physiol. 119A (1998), 717-723

ROSSDALE, P.D.; OUSEY, J.C.; McGLADDERY, A.J.; PRANDI, S.; HOLDSTOCK, N.; GRAINGER, L.;

HOUGHTON, E.:

A retrospective study of increased plasma progestagen concentrations in compromised neonatal foals. Reprod. Fertil. Dev. 7 (1995), 567-575

SERALINI, G.-E.:

Regulation factors of corticosteroid-bindung globulin: Lessons from ontogenesis. Horm. Res. 45 (1996), $192-196$

SIMONETTA, G.; WALZER, D.W.; McMILLEN, I.C.:

Effect of feeding on the diurnal rhythm of plasma cortisol and ACTH concentrations in the pregnant ewe and the sheep fetus. Exp. Physiol. 76 (1991), 219-229

STEINHARDT, M.; BÜNGER, U.; LANGANKE, M.; FIEBIG, U.; GOLLNAST, I.: Geburtsmassen und Maße einiger Körperteile totgeborener Kälber einer großen Milchrindherde. Dtsch. tierärztl. Wschr. 99 (1992), 433-472

STEINHARDT, M.; BÜNGER, U.; LANGANKE, M.; FIEBIG, U.; GOLLNAST, I.: Bemerkungen zum Reifegrad totgeborener Kälber. Tierärztl. Prax. 21 (1993), 201-208

STEINHARDT, M.; THIELSCHER, H.-H.; LEHR, A.; SZALONY, S.; von HORN, T.; DEHN, H.; von HORN, R.; ERMGASSEN, K.; LADEWIG, J.; SMIDT, D.: Verhaltensphysiologische und Belastungsreaktionen bei Rindern verschiedener Rassen und bei ihren Nachkommen im geburtsnahen Zeitraum. Landbauforschung Völkenrode 44 (1994a), 325-339

STEINHARDT, M.; THIELSCHER, H.-H.; von HORN, T.; von HORN, R.; ERMGASSEN, K.; LADEWIG, J.; SMIDT, D.:

Bemerkungen zur Hämoglobinkonzentration des Blutes bei Milchrindern verschiedener Rassen und bei ihren Nachkommen im peripartalen Zeitraum. Tierärztl. Prax. 22 (1994b), 1-7 
STEINHARDT, M.; THIELSCHER, H.-H.; von HORN, T.; von HORN, R.; ERMGASSEN, K.; LADEWIG, J.; SMIDT, D.:

Anpassungsreaktionen von Milchrindkälbern in den ersten Lebenstagen. Einflüsse durch Geburtsverlauf und individuelle Regelbreite. Tierärztl. Prax. 23 (1995), 243-249

STEINHARDT, M.; THIELSCHER, H.-H.; DEHN, H.; von HORN, T.; von HORN, SMIDT, D.:

Hämoglobinkonzentration des Blutes neugeborener Kälber - Einflüsse des Alters des Muttertieres, der Haltungsbedingungen und des Geburtsverlaufes. Landbauforschung Völkenrode 46 (1996) 2, 84-94

STEINHARDT, M.; THIELSCHER, H.-H.:

Tiergerechte Haltung und physiologische Funktionen von Tieren. Entwicklungsqualität von Milchrindkälbern nach der Geburt und frühe postnatale Adaptation der Tiere in Gruppenhaltung mit Tränkeautomatenfütterung. Tierärztl. Umschau 55 (2000) 4,189-198

STOTT, G.H.; REINHARD, E.J.:

Adrenal function and passiv immunity in the dystocial calf. J. Dairy Sci. 61 (1978), 1457-1461

TAKEISHI, M.; SHIBATA, S.; TSUMGARI, S.:

Adrenocorticotropin and cortisol levels in the plasma of bovine fetuses and neonates. Jpn. J. Vet. Sci. 51 (1989), 975-980

VERKERK, G.A.; PHIPPS, A.M.; CARRAGHER, F.; MATTHEWS, L.R.; STELWAGEN; K.:

Characterization of milk cortisol concentration as a measure of short-term stress responses in lactating dairy cows. Anim. Welfare 7 (1998), 77-86

VERMOREL, M.; VERNET, J.; DARDILLAT, C.; SAIDO, ?; DEMIGNE, C.; DAVICCO, CM.-J.:

Energy metabolism and thermoregulation in the newborn calf; effect of calving conditions. Can. J. Anim. Sci. 69 (1989), 113-122

WOUDSTRA, B.R.; de WOLF, B.T.H.M.; SMITS, T.M.; NATHANIELSZ, P.W.; ZIJLSTRA, W.G.; AARNOUDSE, J.G.:

Variability of continuously measured arterial $\mathrm{pH}$ and blood gas values in the near term fetal lamb. Pediatr. Res. 38 (1995), 528-532

WRUTNIAK, C.; CABELLO,G.:

Neonatal changes in plasma cortisol, free and total iodothyronine levels in control and hypotrophic lambs. Reprod. Nutr. Develop. 27 (1987), 945-953

Eingegangen: 01.07.2002

Akzeptiert: 02.08.2002

Anschrift der Verfasser

Dr. habil. MARTIN STEINHARDT,

Dr. HANS-HERMANN THIELSCHER

Institut für Tierzucht und Tierverhalten - FAL

D-23847 Westerau 